\title{
2. Verbände im Kulturbereich
}

\subsection{Museumsverbände national und international}

Verbände im Kulturbereich machen etwa $9 \%$ aller Verbände in Deutschland aus. ${ }^{3}$ Die Bandbreite hierbei reicht von bundesweiten, kulturübergreifenden Einrichtungen - hier zu nennen der Deutsche Kulturrat e.V. oder etwa die Kulturpolitische Gesellschaft e. V. - bis hin zu den spezifischen Sparten, wie der bildenden Kunst, Film und Video, musikalischer und literarischer Bildung und vielen mehr. ${ }^{4}$

Diese Studie nimmt eine Eingrenzung vor und beschränkt sich innerhalb der kulturellen Verbandslandschaft lediglich auf die Museumsverbände, berücksichtigt dabei jedoch nicht nur Deutschland, sondern wirft auch ein Schlaglicht auf internationale Perspektive.

Die nationalen Museumsverbände sind in zwei Wirkungsgebiete zu unterteilen. Hier gesondert zu betrachten sind weitere Museumsorganisationen wie beispielsweise die Landesstellen für nichtstaatliche Museen, auf deren Struktur in dieser Studie nicht weiter eingegangen wird. Die Wirkungsgebiete der Museumsverbände sind zum einen regional, meist auf Ebene der einzelnen Bundesländer, zum anderen überregional. Zum zweiten Bereich zählen beispielsweise die Arbeitsgemeinschaft kirchlicher Museen und Schatzkammern, sowie die sich von den Fachgebieten her oft überschneidenden, wichtigsten überregionalen Museumsverbände in Deutschland: der Deutsche Museumsbund e.V. und das International Council of Museums (ICOM Deutschland e. V.). ${ }^{5}$

Abzugrenzen ist ICOM Deutschland e. V. in folgendem Punkt. Der Verband bildet als größter Museumsfachverband in Deutschland sowie als mitgliederstärkstes Nationalkomitee im internationalen ICOM-Weltverband die Schnittstelle Deutschlands zwischen nationaler und internationaler Museumsarbeit und kann somit als Mittler dieser beiden Aspekte gesehen werden.

Auf internationaler Ebene gibt es neben den verschiedenen ICOM-Nationalkomitees zahlreiche Museum Associations einzelner Länder oder einzelner

3 DGVM, https://www.verbaende.com/hintergruende/studien-statistiken.php, zuletzt aufgerufen am 28.II.2020.

4 Vgl. https://www.bildungsserver.de/Verbaende-Vereinigungen-und-Fachgesellschaften-Kulturelle-Bildung--5428-de.html, zuletzt aufgerufen am 04-12.2020.

5 https://www.smb.museum/museen-einrichtungen/institut-fuer-museumsforschung/partner/, zuletzt aufgerufen am i6.II.2020. 
Sparten. ${ }^{6}$ Was zu fehlen scheint ist jedoch eine weitere übergreifende, allumfassende gemeinsame Plattform. In Europa bietet beispielsweise NEMO, das Netzwerk europäischer Museumsorganisationen,7 den verschiedenen Museumsorganisationen, aber auch einzelnen Museen Unterstützung zum Austausch und Netzwerken.

Zusätzlich zu der großen internationalen Reichweite ist der ICOM-Weltverband die einzige internationale Museumsorganisation, die sich mit museumsethischen Themen beschäftigt.

\subsection{Verbandsstruktur und -politik}

Ein Verband ist laut Bundeszentrale für politische Bildung ein »Zusammenschluss von Personen mit gemeinsamen Interessen zur Verfolgung gemeinsamer Ziele « ${ }^{8}$. ICOM Deutschland e.V. ist ein eingetragener gemeinnütziger Verein. Rechtlich gesehen unterscheidet sich die Organisation von Verbänden und Vereinen nicht, ein Verband ist so gesehen immer auch ein Verein, ${ }^{9}$ wobei Verbände oftmals der beruflichen Fortbildung dienen. Die beiden Begriffe werden im Sprachgebrauch oft gleichbedeutend verwendet. Zudem fallen

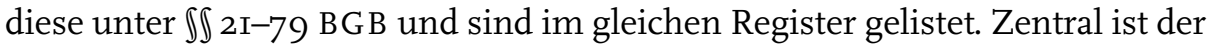
Punkt der Gemeinnützigkeit der Körperschaft nach $\ 52$ Abs. I der Abgabenordnung sowie eine auf Dauer festgelegte Struktur. Konkret bedeutet das, eine von den Gründungsmitgliedern verabschiedete Satzung, und einen in einer Mitgliederversammlung gewählten Vorstand. Es lohnt sich, ICOMs Verbandsstruktur in der Gesamtheit, also auch von der internationalen Perspektive her $\mathrm{zu}$ betrachten.

Der Weltverband mit dem aktuell amtierenden Präsidenten Alberto Garlandini sowie den Vizepräsidenten Terry Simioti Nyambe und Laishun An an der Spitze ${ }^{\mathrm{Io}}$ wurde 1946 in Zusammenarbeit mit der UNESCO gegründet. Die internationale, nichtstaatliche Organisation für Museen gliedert sich in

6 Beispielsweise die International Association of Museums of History.

7 https://www.museumsbund.de/nemo-netzwerk-europaeischer-museumsorganisationen/, zuletzt aufgerufen am 04.0I.2I.

8 https://www.bpb.de/nachschlagen/lexika/politiklexikon/18392/verband-verbaende, zuletzt aufgerufen am 23.05.2I.

9 »Verband« ist kein fester Rechtsbegriff. Nicht jeder Verein ist allerdings umgekehrt auch ein Verband.

10 Durch Wechsel in der Führungsebene im Sommer 2020 wurde Alberto Garlandini als Vizepräsident im Amt des Präsidenten bestätigt. Die Amtszeit läuft aktuell bis zur Generalkonferenz 2022 in Prag. 
die Nationalkomitees der II8 Mitgliedsstaaten. Außerdem gibt es 30 internationale Fachkomitees, die sich schwerpunktmäßig mit bestimmten musealen Themen auseinandersetzen, wie beispielsweise Archäologie und Geschichte, Naturkunde, oder auch mit praktischen Anwendungsgebieten der Museumsarbeit, wie etwa mit der Museumssicherheit. ${ }^{\text {II }}$ Inhaltlich leisten die Komitees zu großen Teilen die Arbeit des Verbandes. Zusätzlich dazu gibt es noch ständige Ausschüsse, Arbeitsgruppen, regionale Allianzen und affiliierte Organisationen. Insgesamt zählt der Weltverband rund 50.000 Mitglieder, ${ }^{12}$ mit etwa 6.500 Mitgliedern aus dem deutschen, dem damit mitgliederstärksten Nationalkomitee. Das zentrale Generalsekretariat des ICOM-Weltverbandes hat seinen Sitz in Paris, Frankreich.

Das deutsche ICOM-Nationalkomitee gründete sich am I9. März I953 und hat seinen Sitz in Berlin. Nach der Wiedervereinigung Deutschlands wurde das Ostdeutsche Nationalkomitee mitaufgenommen. Im Wesentlichen setzt sich der Verband in Deutschland aus den Mitarbeiter*innen der Geschäftsstelle sowie einem gewählten Vorstand bestehend aus sechs Personen und dem gewählten Präsidenten an der Verbandsspitze zusammen. Die aktuell amtierende Präsidentin des deutschen Nationalkomitees ist Frau Prof. Dr. Reifenscheid-Ronnisch, Leiterin des Ludwig Museums in Koblenz. Die Amtszeiten der Repräsentant*innen belaufen sich auf jeweils drei Jahre und ein ICOM-Präsident kann für eine zweite Amtszeit wiedergewählt werden.

Eine solche (Wieder-)Wahl findet im Rahmen interner Mitgliederversammlungen statt. Diese sind Teil der regelmäßig stattfindenden Tagungen auf denen Museumsfachleute, dabei wohl insbesondere die Mitglieder von ICOM Deutschland, über aktuelle museumsrelevante Fragestellungen diskutieren. Die Mitgliederversammlungen finden im Anschluss dazu unter Ausschluss der Öffentlichkeit, und bei anstehenden Neuwahlen mit geheimer, demokratischer Wahl, statt.

Auf internationaler Ebene finden diese Mitgliederversammlungen, die sogenannten Generalversammlungen, alle drei Jahre statt.

11 Internationale Komitees sind beispielsweise COMCOL (International Committee for Collecting), CIDOC (International Committee for Documentation), ICMS (International Committee for Museum Security).

12 Stand 2020. 
